\title{
Reliability of Conclusions from Early Analyses of Real-World Data for Newly Approved Drugs in Advanced Gastric Cancer in the United States
}

This article was published in the following Dove Press journal: Pragmatic and Observational Research

\author{
Lisa $M$ Hess $\mathbb{D}^{\prime}$ \\ Michael Grabner (iD ${ }^{2}$ \\ Liya Wang ${ }^{2}$ \\ Astra M Liepa iD \\ Xiaohong Ivy $\mathrm{Li}^{3}$ \\ Zhanglin Lin Cui ${ }^{3}{ }^{3}$ \\ Lee Bowman iD ' \\ William R Schelman ${ }^{4}$ \\ 'Global Patient Outcomes, Eli Lilly and \\ Company, Indianapolis, IN, USA; ${ }^{2}$ Life \\ Sciences Research, HealthCore Inc., \\ Wilmington, DE, USA; ${ }^{3}$ Global Statistics, \\ Eli Lilly and Company, Indianapolis, IN, \\ USA; ${ }^{4}$ Medical Affairs, Eli Lilly and \\ Company, Indianapolis, IN, USA
}

Background: As real-world data resources expand and improve, there will increasingly be opportunities to study the effectiveness of interventions. There is a need to ensure that study designs explore potential sources of bias and either acknowledge or mitigate them, in order to improve the accuracy of findings. The objective of this study was to understand newly approved drug utilization patterns in real-world clinical settings over time.

Methods: This retrospective study included three sources of real-world data (claims, electronic health records, and recoded data from a quality care program) collected from patients diagnosed with gastric cancer who initiated therapy with either trastuzumab or ramucirumab. Linear regression was used to investigate trends in the use of these drugs for the care of patients with gastric cancer over time from Food and Drug Administration (FDA) approval.

Results: Eligible patients $(n=1700)$ had consistent demographic and clinical characteristics over time. After regulatory approval, trastuzumab was used in later lines of therapy and then shifted to earlier lines $(\mathrm{p}=0.002)$, while ramucirumab utilization remained consistent over time after FDA approval ( $\mathrm{p}=0.49$ ). Ramucirumab augmentation, defined as the addition of the drug after initiation of a line of therapy, decreased over time $(\mathrm{p}=0.03)$, and trastuzumab augmentation remained consistent over time $(\mathrm{p}=0.58)$.

Conclusion: Since treatment effectiveness may change across lines of treatment, bias may arise if there are changes in the use of the drug (such as line migration) during the time period of analysis using real-world data.

Keywords: gastric cancer, trastuzumab, ramucirumab, bias, real-world data

\section{Plain Language Summary}

The process of incorporating new drugs into clinical practice varied in gastric cancer. This study utilized several real-world data sources to investigate trends of utilization of drugs for the care of patients with gastric cancer over time. Trastuzumab migrated from later to earlier lines of therapy over time, although eligible patients had consistent demographic and clinical characteristics, while ramucirumab did not change over time. Such differences in treatment patterns over time or across therapies should be taken into account when estimating comparative effectiveness using real-world data in order to avoid bias. If not considered, bias may arise if there are changes in the use of the drug (such as line migration) during the time period of analysis.

\section{Introduction}

The benefits and risks of health care interventions in a real-world population may not become apparent for some time after approval. ${ }^{1,2}$ Clinical trials that support product registration provide data on the safety and efficacy of an intervention, but
Eli Lilly and Company, Indianapolis, IN 46285, USA

Email Hess_lisa_m@lilly.com 
because less than $1 \%$ of cancer patients participate in trials, they have limited generalizability for the broader population of eligible patients. ${ }^{3}$ This is also a concern with rare diseases, where a limited number of higher-volume clinics may participate in trials but these clinics may not reflect the broader diversity of the patient population. ${ }^{3}$

As a result, comparative effectiveness research and benefit-risk assessment have increasingly turned to the use of big data. Big data sources include a range of real-world databases, such as health care insurance claims and electronic health records (EHR), which contain data collected as part of the routine process of providing and paying for health care. Increasingly, researchers are using big data to enhance the ability to understand how drugs, procedures or other interventions perform in non-experimental, realworld conditions (ie, outside of a controlled research setting). ${ }^{4}$ These datasets can provide the ability to answer certain research questions in a timely and less costly manner than a prospective clinical trial, and may help to understand the need for or to inform the development of randomized trials. ${ }^{5}$ Additionally, larger databases enable the study of smaller subgroups, or patients with rare comorbidities or conditions, that may not have participated in the clinical trial in sufficient numbers, and thereby may support the exploration of patient populations that may ultimately benefit the most from the new treatment or intervention. ${ }^{6}$

These real-world data are collected in real time and therefore may enable knowledge of the risks and benefits to become evident sooner than would have taken place with a systematic or prospective study. A key objective for retrospective analyses of a new drug using big data is to reveal the pattern by which the new drug is incorporated into routine clinical practice. It is fundamentally important that the observed outcomes reflect the true real-world utilization and are not an artifact of a biased trial sample. ${ }^{7}$ For example, health care providers may be cautious about a new drug and use it in only the healthiest patients. Alternatively, a newly approved drug may be used in refractory patients due to the lack of other viable treatment options. This is of particular concern in a disease with high unmet need. As a result, there could be an overestimate of benefit and underestimate of risk compared to other therapies or no treatment, or vice versa, until providers become familiar with the drug and are better able to identify the optimal patient for the new treatment. This potential bias should be considered at the study design stage, particularly if the analysis is conducted shortly after the approval of a new drug.
A number of treatment options are being developed for gastric cancer, for which there have been few approved drugs over the past decade. In October 2010, trastuzumab (Genentech, San Francisco, CA, USA) received an FDA approval for use as first-line treatment in combination with a fluoropyrimidine and cisplatin in patients with HER2overexpressing metastatic gastric or gastroesophageal junction (GEJ) adenocarcinoma, in addition to its existing approval for the treatment of patients with HER2-overexpressing breast cancer from September 1998. In April 2014, ramucirumab (Eli Lilly and Company, Indianapolis, IN, USA) was first approved as a single agent by the FDA for the treatment of patients with advanced gastric or GEJ adenocarcinoma after prior fluoropyrimidine- or platinum-containing chemotherapy, and in November 2014 was approved in the same setting in combination with paclitaxel. These agents were selected for study as they were the newest agents that received FDA approval in the setting of gastric cancer at the time of this study. While clinical trial data demonstrate the efficacy of these drugs, it is important for manufacturers, providers, payers, and patients to understand the effectiveness of new drugs in routine practice, and to compare their outcomes against other recommended therapies. Given the small patient population and the high variability in treatment of gastric and GEJ cancers, it is not feasible to expect a series of randomized trials to be conducted against all relevant treatment options. The ability to conduct comparative effectiveness research to answer these important questions may rely on claims or EHR data.

This study investigated the potential risk of bias due to changes in how providers select patients for new therapies shortly after their initial FDA approval. The specific objectives were to characterize the demographic and clinical characteristics of patients receiving trastuzumab or ramucirumab in the initial years after their respective approvals in these indications, and to compare the treatment patterns and patient populations over time.

\section{Methods}

A retrospective cohort study evaluated longitudinal pharmacy and medical data from three sources: claims data from the HealthCore Integrated Research Database; clinical data from a Cancer Care Quality Program contained in the HealthCore Integrated Research Environment; and EHR data from the Flatiron Health Advanced Gastric/ Esophageal Cancer Cohort. 


\section{HealthCore Integrated Research \\ Database (HIRD)}

The HIRD contains administrative claims data from 14 health plans in the Northeast, Midwest, South and West regions of the US starting in January 2006 and represents claims information from one of the largest commercially insured populations in the US. As with all claims data sources, limited clinical variables are collected. The HIRD contains data for both trastuzumab and ramucirumab.

\section{Cancer Care Quality Program (CCQP)}

The CCQP utilizes cancer treatment pathways, selected based upon current clinical evidence, published literature, and national guideline recommendations. Evidence-based quality care is supported via additional reimbursement per patient for prescribed treatment regimens that align with the identified pathway. Data are entered into the CCQP system when physicians request approval for this pathwaybased enhanced reimbursement as well as prior authorization for various cancer treatments. This program has been implemented in all of the health plans represented in the HealthCore Integrated Research Environment since September 2015. As the CCQP was implemented several years after the approval of trastuzumab, only ramucirumab could be evaluated using this data source.

\section{Flatiron Health Electronic Health Records (EHR)}

The Flatiron Health Advanced Gastric/Esophageal Cancer Cohort is a subset of the overall Flatiron Health Database that includes a geographically diverse sample of over 7500 patients with advanced gastric/GEJ/esophageal cancer at community oncology and academic cancer centers in the US. The database includes patients whose advanced cancer diagnoses occurred on or after January 1, 2011 and who have two or more visits documented in the EHR during that time period. Due to the lack of data prior to 2011, trastuzumab could not be studied in this database, as data are not available at and following its initial approval in gastric cancer.

\section{Eligibility Criteria}

Eligible patients in the HIRD were age 18 or older with a diagnosis of gastric or GEJ cancer (ICD-9-CM code 151. $\mathrm{xx}$; ICD-10-CM codes: C16.x). The trastuzumab claims cohort included patients who received trastuzumab at any time between January 1, 2010 and March 31, 2017 (to allow for follow-up data until July 31, 2017, the most recently available data at the time of the analysis). While trastuzumab was approved in October of 2010, January was used as the start date as the drug was FDA approved for other indications, and the clinical trial results were published in $2009,{ }^{8}$ therefore, any early users would have been missed with a later start period. Patients were ineligible for the trastuzumab cohort if they received trastuzumab prior to 2010 or if they were diagnosed with breast cancer at any time. Patients were eligible for the ramucirumab claims cohort if they received ramucirumab between January 1, 2014 and March 31, 2017, and were ineligible if they received ramucirumab prior to 2014 or if they were diagnosed at any time with lung or colorectal cancer (ramucirumab was subsequently approved for use by the FDA in these tumor types during the study period). Of note, ramucirumab did not have a Healthcare Common Procedure Coding System (HCPCS) J-code assigned until January 1, 2016. C9025 was a temporary HCPCS code in effect between October 1, 2014 and December 31, 2015 and was replaced by J9308 on January 1, 2016 as the permanent HCPCS codes for ramucirumab. Therefore, HCPCS codes C9025 and J9308 were used in combination with National Drug Code (NDC) and Generic Product Identifier (GPI) codes to identify ramucirumab in claims.

Eligibility criteria for the CCQP cohort and the Flatiron EHR cohort were similar; patients were age 18 or older with a diagnosis of gastric, GEJ or esophageal adenocarcinoma who received ramucirumab. These criteria were evaluated from April 21, 2014 through March 31, 2018 for the EHR and from June 23, 2014 through April 13, 2018 for CCQP, based on data completeness and availability of datasets. A complete trastuzumab cohort could not be identified in either the CCQP or EHR data due to the lack of data coinciding with the time of FDA approval for gastric/GEJ adenocarcinoma. Therefore, four cohorts from three data sources were included in this study: trastuzumab claims; ramucirumab claims; ramucirumab CCQP data; and ramucirumab EHR data.

\section{Definition of Treatment Regimens}

The first observed claim or infusion for trastuzumab or ramucirumab was set as the index date in each database, respectively. Descriptive analyses were conducted for each annual cohort of patients receiving trastuzumab as defined by calendar year of index date, as well as for all patients combined. For the ramucirumab cohorts, the time periods were divided into half-year increments (except for the CCQP cohort, which used annual periods) due to the shorter 
duration of follow up since the date of FDA approval. Treatment regimens were defined by the chemotherapy, biologic and/or targeted agents received, including route of administration (intravenous versus oral), within 28 days of the start of therapy.

Fluoropyrimidine agents (capecitabine and 5-FU) were considered to be equivalent as were the platinum agents of carboplatin and cisplatin. The initial therapy received after the first evidence of advanced gastric/GEJ cancer diagnosis was defined as the first line of therapy. A line of therapy ended when either the patient discontinued all treatment for 42 days or more, or the patient added a new drug to the regimen, while maintaining at least one of the initial regimen drugs. Removal of some, but not all, drugs in a regimen did not constitute a change in line of therapy. The date of the addition of the new agent was considered the start of the next line of therapy, with the exception of the addition of a biologic or targeted agent to the chemotherapy regimen, which was considered augmentation and did not advance the line of therapy the patient was receiving.

\section{Statistical Analysis}

Descriptive statistics included mean, standard deviation (SD), median, and range for continuous variables, and frequency count and percentage for categorical variables.

\section{Claims data}

Statistical testing of patient characteristics across the time periods (annual for trastuzumab and the CCQP ramucirumab cohort, bi-annual for all other ramucirumab cohorts) was conducted using a chi-squared or Fisher's exact test for categorical variables and F-test for continuous variables. Linear regression was used to investigate trends in trastuzumab use over time (percent of patients using trastuzumab in first line in claims). Post-hoc testing for the time from initial diagnosis to first chemotherapy in claims was conducted using Tukey's honestly significant difference (HSD) test, in order to explore which years or halfyears were significantly different from each other while accounting for multiple pairwise comparisons. The duration of treatment was calculated in days with a restricted mean survival time (RMST) and SD and compared over time using a Log-rank test. The treatment duration time period was defined as the difference between the date of the first infusion to the date of the last infusion plus 1 day. An alpha level of $<0.05$ was defined to identify statistical significance. All analyses were conducted using SAS version 9.4 (SAS Institute Inc., Cary, NC, USA).

\section{Results}

A total of 1700 patients were identified across the four study cohorts (Figure 1). Table 1 provides the demographic and

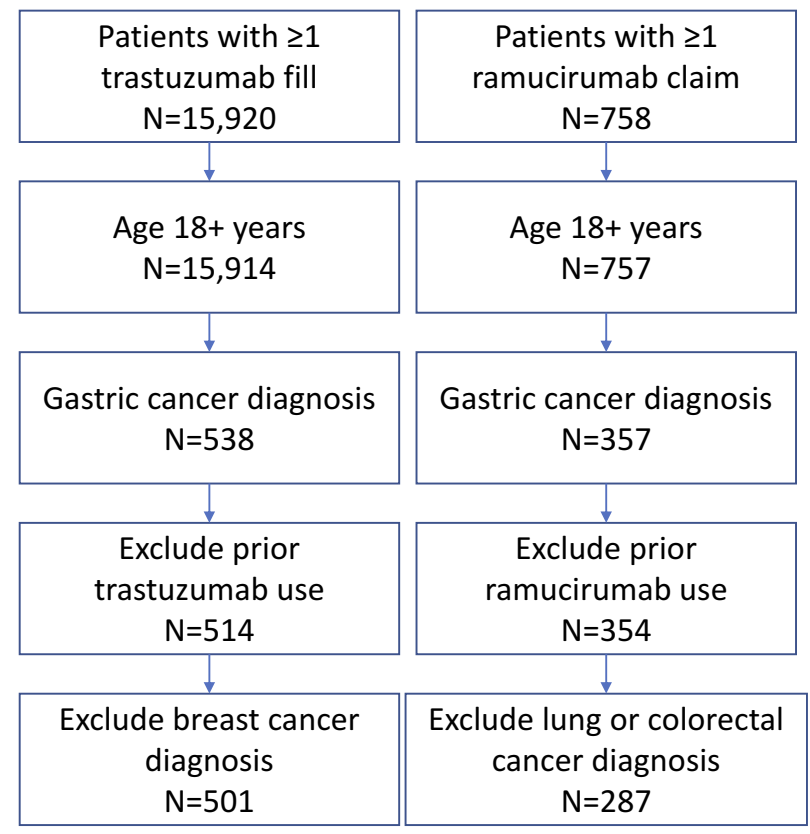

CCQP=Cancer Care Quality Program; GEJ=gastroesophageal junction

\section{CCQP data}

Patients with $\geq 1$ preauthorization for ramucirumab $\mathrm{N}=912$

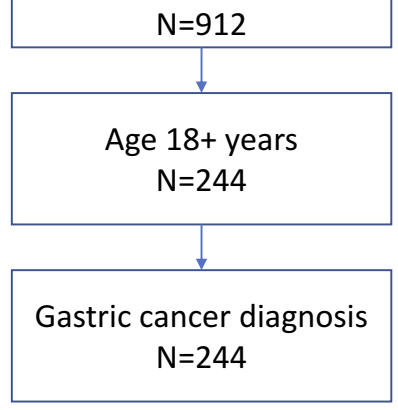$$
\text { . }
$$

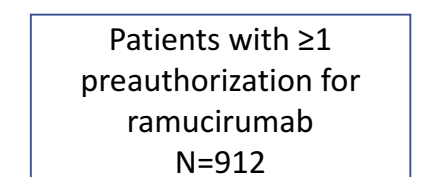

Figure I Flow chart of patient eligibility.

\section{Electronic health records}

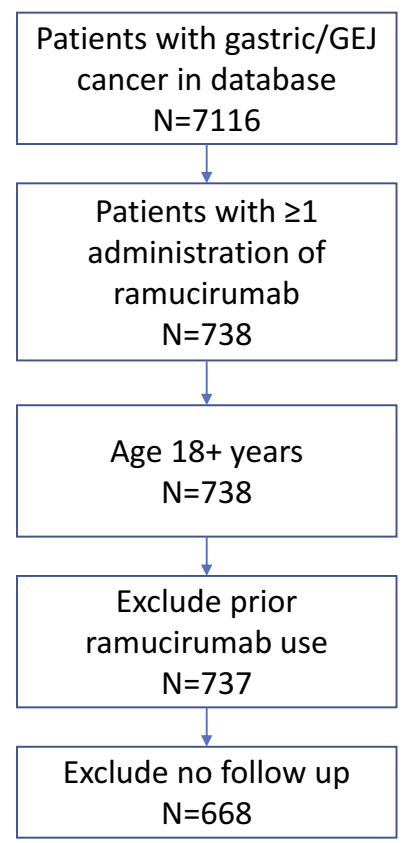


Table I Trastuzumab Claims Cohort, Overall and by Index Year

\begin{tabular}{|c|c|c|c|c|c|c|c|c|c|c|}
\hline \multicolumn{11}{|c|}{ Demographic and Clinical Characteristics } \\
\hline & Overall & 2010 & 2011 & 2012 & 2013 & 2014 & 2015 & 2016 & 2017 & p-value ${ }^{a}$ \\
\hline $\mathbf{N}$ & 501 & 24 & 51 & 49 & 76 & 84 & 82 & 87 & 48 & \\
\hline \multicolumn{11}{|c|}{ Age at index date in years, } \\
\hline mean (SD) & $61.1(11.7)$ & $59.3(11.6)$ & $60.2(10.8)$ & $61.9(10.5)$ & $60.9(12.4)$ & $61.8(12.9)$ & $61.9(11.0)$ & $62.0(11.5)$ & $58.5(12.3)$ & 0.70 \\
\hline \multicolumn{11}{|l|}{ Gender, $\mathbf{n}(\%)$} \\
\hline Female & $95(19.0)$ & $4(16.7)$ & $9(17.6)$ & $9(18.4)$ & II (14.5) & $13(15.5)$ & $16(19.5)$ & $19(21.8)$ & $14(29.2)$ & 0.59 \\
\hline Male & $406(81.0)$ & $20(83.3)$ & $42(82.4)$ & $40(81.6)$ & $65(85.5)$ & $71(84.5)$ & $66(80.5)$ & $68(78.2)$ & $34(70.8)$ & \\
\hline \multicolumn{11}{|l|}{ Health plan type, $n$ (\%) } \\
\hline HMO & $108(21.6)$ & $6(25.0)$ & $12(23.5)$ & $6(12.2)$ & $16(21.1)$ & $15(17.9)$ & $20(24.4)$ & $22(25.3)$ & II (22.9) & 0.70 \\
\hline PPO & $34 I(68.1)$ & $17(70.8)$ & $37(72.5)$ & $34(69.4)$ & $52(68.4)$ & $60(71.4)$ & $57(69.5)$ & $54(62.1)$ & $30(62.5)$ & 0.86 \\
\hline $\mathrm{CDHP}$ & $51(10.2)$ & I (4.2) & $2(3.9)$ & $9(18.4)$ & $8(10.5)$ & $9(10.7)$ & $4(4.9)$ & $11(12.6)$ & $7(14.6)$ & 0.14 \\
\hline Other & $\mathrm{I}(0.2)$ & $0(0)$ & $0(0)$ & $0(0)$ & $0(0)$ & $0(0)$ & $\mathrm{I}(1.2)$ & $0(0)$ & $0(0)$ & 0.66 \\
\hline \multicolumn{11}{|c|}{ Non-commercial enrollment, $\mathbf{n}(\%)$} \\
\hline $\begin{array}{l}\text { Medicare Advantage, } \\
\text { Supplemental, or Part D }\end{array}$ & $119(23.8)$ & $7(29.2)$ & $14(27.5)$ & $19(38.8)$ & $22(28.9)$ & $17(20.2)$ & $20(24.4)$ & $14(16.1)$ & $6(12.5)$ & 0.04 \\
\hline \multicolumn{11}{|l|}{ Geographic region, n (\%) } \\
\hline Northeast & $93(18.6)$ & $4(16.7)$ & $6(11.8)$ & $8(16.3)$ & $19(25.0)$ & $12(14.3)$ & $10(12.2)$ & $23(26.4)$ & II (22.9) & 0.13 \\
\hline Midwest & $143(28.5)$ & $8(33.3)$ & $16(31.4)$ & $15(30.6)$ & $23(30.3)$ & $29(34.5)$ & $22(26.8)$ & $20(23.0)$ & $10(20.8)$ & 0.66 \\
\hline South & $136(27.1)$ & $6(25.0)$ & $7(13.7)$ & $15(30.6)$ & $17(22.4)$ & $23(27.4)$ & $28(34.1)$ & $25(28.7)$ & $15(31.3)$ & 0.30 \\
\hline West & $129(25.7)$ & $6(25.0)$ & $22(43.1)$ & II (22.4) & $17(22.4)$ & $20(23.8)$ & $22(26.8)$ & $19(21.8)$ & $12(25.0)$ & 0.20 \\
\hline \multicolumn{11}{|c|}{ Site of care at index infusion, $n(\%)$} \\
\hline Physician office visit & $236(47.1)$ & $15(62.5)$ & $28(54.9)$ & $23(46.9)$ & $36(47.4)$ & $40(47.6)$ & $23(28.0)$ & $49(56.3)$ & $22(45.8)$ & 0.01 \\
\hline Outpatient hospital & $254(50.7)$ & $7(29.2)$ & $22(43.1)$ & $25(51.0)$ & $38(50.0)$ & $43(51.2)$ & $57(69.5)$ & $36(41.4)$ & $26(54.2)$ & 0.005 \\
\hline Inpatient hospital/ER & $\mathrm{I}(0.2)$ & $0(0)$ & $0(0)$ & $I(2.0)$ & $0(0)$ & $0(0)$ & $0(0)$ & $0(0)$ & $0(0)$ & 0.24 \\
\hline All other locations & $10(2.0)$ & $2(8.3)$ & I (2.0) & $0(0)$ & $2(2.6)$ & I ( 1.2$)$ & $2(2.4)$ & $2(2.3)$ & $0(0)$ & 0.47 \\
\hline \multicolumn{11}{|c|}{ Time from initial diagnosis to index infusion, days } \\
\hline Median (IQR) & $\begin{array}{l}47.0 \\
(126.0)\end{array}$ & $\begin{array}{l}207.0 \\
(205.5)\end{array}$ & $\begin{array}{l}57.0 \\
(172.0)\end{array}$ & $\begin{array}{l}76.0 \\
(194.0)\end{array}$ & $\begin{array}{l}54.0 \\
(96.5)\end{array}$ & $\begin{array}{l}41.5 \\
(139.5)\end{array}$ & $\begin{array}{l}41.0 \\
(62.0)\end{array}$ & $\begin{array}{l}46.0 \\
(76.0)\end{array}$ & $\begin{array}{l}41.5 \\
(69.5)\end{array}$ & \\
\hline \multirow[t]{2}{*}{ Mean (SD) } & 96.2 & 167.8 & 117.1 & 130.5 & 92.2 & 95.9 & 75.1 & 79.6 & 76.2 & 0.002 \\
\hline & $(113.8)$ & $(139.3)$ & $(123.13)$ & $(132.8)$ & $(96.1)$ & $(117.2)$ & $(105.6)$ & $(96.1)$ & $(113.7)$ & \\
\hline \multicolumn{11}{|c|}{ Follow up time period, days } \\
\hline \multirow[t]{2}{*}{ Median (IQR) } & 273.0 & 231.0 & 234.0 & 258.0 & 286.5 & 326.0 & 362.0 & 238.0 & 220.5 & \\
\hline & $(349.0)$ & $(422.0)$ & $(344.0)$ & $(4 \mid 1.0)$ & $(4 \mid 4.5)$ & $(523.0)$ & $(498.0)$ & $(317.0)$ & $(139.0)$ & \\
\hline \multirow[t]{2}{*}{ Mean (SD) } & 368.1 & 390.0 & 360.4 & 393.7 & 399.4 & 420.2 & 433.9 & 295.0 & 218.2 & 0.004 \\
\hline & $(329.1)$ & $(430.0)$ & $(355.5)$ & $(401.6)$ & $(391.3)$ & $(354.1)$ & $(309.8)$ & $(203.2)$ & $(90.8)$ & \\
\hline \multicolumn{11}{|c|}{ Quan Charlson Comorbidity Items, n (\%) } \\
\hline Myocardial infarction & $22(4.4)$ & $0(0)$ & $4(7.8)$ & $I(2.0)$ & $\mathrm{I}(\mathrm{I} .3)$ & $9(10.7)$ & $3(3.7)$ & $\mathrm{I}(\mathrm{l} . \mathrm{I})$ & $3(6.3)$ & 0.04 \\
\hline Congestive heart failure & $48(9.6)$ & I (4.2) & $6(11.8)$ & $3(6.1)$ & $7(9.2)$ & $7(8.3)$ & $12(14.6)$ & $9(10.3)$ & $3(6.3)$ & $\mathrm{NE}$ \\
\hline $\begin{array}{l}\text { Peripheral vascular } \\
\text { disease }\end{array}$ & $68(13.6)$ & I (4.2) & $7(13.7)$ & $2(4.1)$ & $10(13.2)$ & II (13.1) & $14(17.1)$ & $14(16.1)$ & $9(18.8)$ & 0.33 \\
\hline Cerebrovascular disease & $45(9.0)$ & $0(0)$ & $2(3.9)$ & $6(12.2)$ & II (14.5) & $8(9.5)$ & $9(11.0)$ & $6(6.9)$ & $3(6.3)$ & $N E$ \\
\hline Dementia & $2(0.4)$ & $0(0)$ & $0(0)$ & $I(2.0)$ & $0(0)$ & $0(0)$ & $\mathrm{I}(1.2)$ & $0(0)$ & $0(0)$ & 0.44 \\
\hline $\begin{array}{l}\text { Chronic pulmonary } \\
\text { disease }\end{array}$ & $101(20.2)$ & $2(8.3)$ & $14(27.5)$ & $9(18.4)$ & $13(17.1)$ & $17(20.2)$ & $20(24.4)$ & $16(18.4)$ & $10(20.8)$ & 0.61 \\
\hline
\end{tabular}


Table I (Continued).

\begin{tabular}{|c|c|c|c|c|c|c|c|c|c|c|}
\hline \multicolumn{11}{|c|}{ Demographic and Clinical Characteristics } \\
\hline & Overall & 2010 & 2011 & 2012 & 2013 & 2014 & 2015 & 2016 & 2017 & p-value ${ }^{a}$ \\
\hline $\mathbf{N}$ & 501 & 24 & 51 & 49 & 76 & 84 & 82 & 87 & 48 & \\
\hline $\begin{array}{l}\text { Connective tissue/ } \\
\text { rheumatic disease }\end{array}$ & $5(1.0)$ & $0(0)$ & $0(0)$ & $0(0)$ & I (I.3) & $2(2.4)$ & $0(0)$ & $I(I .1)$ & I (2.1) & 0.80 \\
\hline $\begin{array}{l}\text { Peptic ulcer disease } \\
\text { Liver disease }\end{array}$ & $57(11.4)$ & $2(8.3)$ & $9(17.6)$ & $4(8.2)$ & $8(10.5)$ & $9(10.7)$ & $8(9.8)$ & $12(13.8)$ & $5(10.4)$ & 0.83 \\
\hline Mild & $165(32.9)$ & $4(16.7)$ & $18(35.3)$ & $19(38.8)$ & $27(35.5)$ & $25(29.8)$ & $26(31.7)$ & $27(31.0)$ & $19(39.6)$ & 0.60 \\
\hline Moderate or severe & $4(0.8)$ & $0(0)$ & $2(3.9)$ & $\mathrm{I}(2.0)$ & $0(0)$ & $0(0)$ & $0(0)$ & $\mathrm{I}(1.1)$ & $0(0)$ & 0.17 \\
\hline $\begin{array}{l}\text { Paraplegia and } \\
\text { hemiplegia }\end{array}$ & $3(0.6)$ & $0(0)$ & $2(3.9)$ & $0(0)$ & $0(0)$ & $0(0)$ & $0(0)$ & $\mathrm{I}(\mathrm{I.1})$ & $0(0)$ & 0.18 \\
\hline $\begin{array}{l}\text { Renal disease } \\
\text { Diabetes }\end{array}$ & $27(5.4)$ & $2(8.3)$ & $0(0)$ & I (2.0) & $8(10.5)$ & $7(8.3)$ & $4(4.9)$ & $4(4.6)$ & I (2.1) & NE \\
\hline $\begin{array}{l}\text { Diabetes without } \\
\text { chronic complications }\end{array}$ & III (22.2) & $4(16.7)$ & II (21.6) & $13(26.5)$ & $20(26.3)$ & $19(22.6)$ & $18(22.0)$ & $15(17.2)$ & II (22.9) & 0.89 \\
\hline $\begin{array}{l}\text { Diabetes with chronic } \\
\text { complications }\end{array}$ & $18(3.6)$ & $0(0)$ & $3(5.9)$ & $0(0)$ & $3(3.9)$ & $3(3.6)$ & $2(2.4)$ & $4(4.6)$ & $3(6.3)$ & 0.68 \\
\hline Cancer & $410(81.8)$ & $22(91.7)$ & $41(80.4)$ & $45(91.8)$ & $63(82.9)$ & $68(81.0)$ & $62(75.6)$ & $71(81.6)$ & $38(79.2)$ & 0.39 \\
\hline Metastatic carcinoma & $407(81.2)$ & $20(83.3)$ & $42(82.4)$ & $43(87.8)$ & $63(82.9)$ & $63(75.0)$ & $64(78.0)$ & $70(80.5)$ & $42(87.5)$ & 0.59 \\
\hline AIDS/HIV & $2(0.4)$ & $0(0)$ & $0(0)$ & I (2.0) & $\mathrm{I}(1.3)$ & $0(0)$ & $0(0)$ & $0(0)$ & $0(0)$ & 0.32 \\
\hline $\begin{array}{l}\text { Quan Charlson } \\
\text { Comorbidity Index, } \\
\text { Mean (SD) }\end{array}$ & $8.0(3.1)$ & $7.6(2.8)$ & $8.3(3.5)$ & $8.5(2.3)$ & $8.3(3.4)$ & $7.6(3.4)$ & $7.7(3.3)$ & $7.9(2.9)$ & $8.3(2.7)$ & 0.61 \\
\hline \multicolumn{11}{|c|}{ Procedures during 6-month baseline period, $n(\%)$} \\
\hline $\begin{array}{l}\text { Surgery (resection; } \\
\text { gastrectomy) }\end{array}$ & $99(19.8)$ & $2(8.3)$ & $6(11.8)$ & $7(14.3)$ & $14(18.4)$ & $13(15.5)$ & $12(14.6)$ & $28(32.2)$ & $17(35.4)$ & 0.002 \\
\hline Radiation & $129(25.7)$ & $8(33.3)$ & $16(31.4)$ & II (22.4) & $16(21.1)$ & $20(23.8)$ & $22(26.8)$ & $26(29.9)$ & $10(20.8)$ & 0.74 \\
\hline HER2 testing & $321(64.1)$ & $12(50.0)$ & $33(64.7)$ & $30(61.2)$ & $53(69.7)$ & $53(63.1)$ & $46(56.1)$ & $63(72.4)$ & $31(64.6)$ & 0.31 \\
\hline \multicolumn{11}{|l|}{ Treatment patterns } \\
\hline \multicolumn{11}{|c|}{ Line of therapy in which trastuzumab was initiated, $\mathbf{n}(\%)$} \\
\hline First line & $353(70.5)$ & $12(50.0)$ & $30(58.8)$ & $31(63.3)$ & $56(73.7)$ & $58(69.0)$ & $59(72.0)$ & $71(81.6)$ & $36(75.0)$ & 0.03 \\
\hline Second line & $105(21.0)$ & $4(16.7)$ & $14(27.5)$ & $9(18.4)$ & $18(23.7)$ & $20(23.8)$ & $18(22.0)$ & $13(14.9)$ & $9(18.8)$ & 0.71 \\
\hline Third line & $32(6.4)$ & $4(16.7)$ & $7(13.7)$ & $6(12.2)$ & $2(2.6)$ & $4(4.8)$ & $4(4.9)$ & $3(3.4)$ & $2(4.2)$ & $N E$ \\
\hline Fourth line & $6(1.2)$ & $2(8.3)$ & $0(0)$ & $0(0)$ & $0(0)$ & $2(2.4)$ & I (I.2) & $0(0)$ & I (2.I) & NE \\
\hline Fifth line & $4(0.8)$ & I (4.2) & $0(0)$ & $3(6.1)$ & $0(0)$ & $0(0)$ & $0(0)$ & $0(0)$ & $0(0)$ & NE \\
\hline Sixth line & $I(0.2)$ & I (4.2) & $0(0)$ & $0(0)$ & $0(0)$ & $0(0)$ & $0(0)$ & $0(0)$ & $0(0)$ & NE \\
\hline \multicolumn{10}{|c|}{ Regimen in which trastuzumab was initiated ${ }^{\mathrm{c}}, \mathbf{n}(\%)$} & NE \\
\hline Trastuzumab+FOLFOX & $115(23.0)$ & $0(0)$ & $12(23.5)$ & $5(10.2)$ & $13(17.1)$ & $22(26.2)$ & $18(22.0)$ & $27(31.0)$ & $18(37.5)$ & \\
\hline Trastuzumab only & $81(16.2)$ & $4(16.7)$ & $7(13.7)$ & $8(16.3)$ & $14(18.4)$ & $15(17.9)$ & $16(19.5)$ & $13(14.9)$ & $4(8.3)$ & \\
\hline $\begin{array}{l}\text { Trastuzumab+platinum+ } \\
\text { fluoropyrimidine }\end{array}$ & $55(11.0)$ & $4(16.7)$ & $8(15.7)$ & $8(16.3)$ & II (14.5) & $5(6.0)$ & $4(4.9)$ & II (12.6) & $4(8.3)$ & \\
\hline FOLFOX & $47(9.4)$ & $0(0)$ & I (2.0) & $4(8.2)$ & $10(13.2)$ & $6(7.1)$ & $9(11.0)$ & $10(11.5)$ & $7(14.6)$ & \\
\hline Trastuzumab+platinum & $34(6.8)$ & $0(0)$ & $5(9.8)$ & $4(8.2)$ & $6(7.9)$ & $6(7.1)$ & $4(4.9)$ & $6(6.9)$ & $3(6.3)$ & \\
\hline Trastuzumab+oxaliplatin & $15(3.0)$ & $0(0)$ & I (2.0) & $3(6.1)$ & $I(1.3)$ & $2(2.4)$ & $3(3.7)$ & $2(2.3)$ & $3(6.3)$ & \\
\hline $\begin{array}{l}\text { Trastuzumab+platinum+ } \\
\text { paclitaxel }\end{array}$ & $15(3.0)$ & I (4.2) & $3(5.9)$ & $0(0)$ & $4(5.3)$ & $7(8.3)$ & $0(0)$ & $0(0)$ & $0(0)$ & \\
\hline $\begin{array}{l}\text { Platinum }+ \\
\text { fluoropyrimidine }\end{array}$ & $14(2.8)$ & $2(8.3)$ & $0(0)$ & I (2.0) & I (1.3) & $2(2.4)$ & $2(2.4)$ & $4(4.6)$ & $2(4.2)$ & \\
\hline Platinum & $12(2.4)$ & $2(8.3)$ & $0(0)$ & $0(0)$ & $\mathrm{I}(\mathrm{I} .3)$ & $\mathrm{I}(\mathrm{I} .2)$ & $4(4.9)$ & $3(3.4)$ & I (2.1) & \\
\hline
\end{tabular}

(Continued) 
Table I (Continued).

\begin{tabular}{|c|c|c|c|c|c|c|c|c|c|c|}
\hline \multicolumn{11}{|c|}{ Demographic and Clinical Characteristics } \\
\hline & Overall & 2010 & 2011 & 2012 & 2013 & 2014 & 2015 & 2016 & 2017 & p-value ${ }^{a}$ \\
\hline $\mathbf{N}$ & 501 & 24 & 51 & 49 & 76 & 84 & 82 & 87 & 48 & \\
\hline Platinum+paclitaxel & $10(2.0)$ & $0(0)$ & $2(3.9)$ & $4(8.2)$ & $2(2.6)$ & I (1.2) & I (I.2) & $0(0)$ & $0(0)$ & \\
\hline Trastuzumab+irinotecan & $9(1.8)$ & $2(8.3)$ & $0(0)$ & I (2.0) & $2(2.6)$ & $\mathrm{I}(\mathrm{I} .2)$ & $\mathrm{I}(\mathrm{I} .2)$ & $2(2.3)$ & $0(0)$ & \\
\hline Fluoropyrimidine & $6(1.2)$ & $0(0)$ & $2(3.9)$ & $0(0)$ & $0(0)$ & $2(2.4)$ & $0(0)$ & $\mathrm{I}(\mathrm{I.I})$ & I (2.1) & \\
\hline Oxaliplatin+epirubicin+ & $6(1.2)$ & $0(0)$ & I (2.0) & I (2.0) & $0(0)$ & $0(0)$ & $2(2.4)$ & I (I.I) & I (2.1) & \\
\hline fluoropyrimidine & & & & & & & & & & \\
\hline Trastuzumab+oxaliplatin & $6(1.2)$ & $0(0)$ & $0(0)$ & $0(0)$ & $2(2.6)$ & I (1.2) & $3(3.7)$ & $0(0)$ & $0(0)$ & \\
\hline + epirubicin+ & & & & & & & & & & \\
\hline fluoropyrimidine & & & & & & & & & & \\
\hline Trastuzumab+paclitaxel & $6(1.2)$ & I (4.2) & I (2.0) & I (2.0) & $0(0)$ & $3(3.6)$ & $0(0)$ & $0(0)$ & $0(0)$ & \\
\hline Oxaliplatin & $5(1.0)$ & I (4.2) & $0(0)$ & $0(0)$ & I (1.3) & $0(0)$ & $3(3.7)$ & $0(0)$ & $0(0)$ & \\
\hline Fluoropyrimidine & $4(0.8)$ & $2(8.3)$ & $0(0)$ & $0(0)$ & $0(0)$ & $0(0)$ & $0(0)$ & $0(0)$ & $2(4.2)$ & \\
\hline +irinotecan & & & & & & & & & & \\
\hline Augmentation with & $121(24.2)$ & $8(33.3)$ & $9(17.6)$ & $14(28.6)$ & $19(25.0)$ & $15(17.9)$ & $22(26.8)$ & $20(23.0)$ & $14(29.2)$ & 0.58 \\
\hline trastuzumab $^{\mathrm{d}}, \mathbf{n}(\%)$ & & & & & & & & & & \\
\hline \multicolumn{11}{|c|}{ Duration of trastuzumab therapy, days } \\
\hline \multirow[t]{2}{*}{ RMST (SD) } & 403.6 & 393.0 & 386.7 & 374.2 & 379.5 & 369.4 & 312.9 & 268.1 & 157.4 & 0.99 \\
\hline & (27.8) & (I $\mid 8.3)$ & $(69.4)$ & (59.1) & $(47.5)$ & (46.5) & (29.7) & $(24.0)$ & (13.1) & \\
\hline
\end{tabular}

Notes: ${ }^{\mathrm{a}} \mathrm{P}$-values were based on $\mathrm{p}$-values were based on F-test for continuous variables and Chi-squared/Fisher-exact test for categorical variables. Duration of trastuzumab therapy was compared using a Log-rank test. ${ }^{b}$ Gastric cancer codes (International Classification of Disease, ICD-9-CM, clinical modification: I5I; ICD-I0-CM: CI6) were excluded from the standard Quan Charlson Comorbidity Index coding algorithm in identifying cancer conditions. ${ }^{\mathrm{C}}$ Limited to regimens with $\mathrm{n}>\mathrm{I}$. ${ }^{\mathrm{d}}$ Trastuzumab was added to the chemotherapy regimen. Bolded text represents column headers and $p$-values $<0.05$.

Abbreviations: HMO, health maintenance organization; PPO, preferred provider organization; CDHP, consumer-directed health plan; SD, standard deviation, IQR, interquartile range; AIDS/HIV, acquired immune deficiency syndrome/human immunodeficiency virus; HER2, human epidermal growth factor receptor 2; ER, emergency room; FOLFOX, fluoropyrimidine + oxaliplatin; NE, not estimable; RMST, restricted mean survival time.

clinical characteristics of the 501 eligible patients in the trastuzumab claims cohort overall as well as by year of initiation of trastuzumab. Table 2 provides characteristics of the ramucirumab claims cohort $(n=287)$; characteristics of the ramucirumab CCQP $(n=244)$ and EHR cohorts $(n=668)$ are in Tables 3 and 4. Patients were on average $61,60,57$ and 64 years of age in the trastuzumab claims, ramucirumab claims, ramucirumab $\mathrm{CCQP}$ and ramucirumab EHR cohorts, respectively. In all datasets, most patients were male. Demographic characteristics were relatively consistent across date of trastuzumab or ramucirumab initiation in all cohorts (Tables 1-4). Similar to trends in oncology in general, ${ }^{9,10}$ the place of infusion shifted over time from the physician office to the outpatient hospital setting across the claims dataset. Additionally, the composition of insurance plan types was statistically different during the time periods evaluated. The proportion of patients receiving trastuzumab as first line was significantly different during the periods evaluated (Table 1); for ramucirumab (Tables 2 and 3), utilization seemed to move from fourth and higher lines into both first- and second-line therapy. The duration of therapy did not vary over the time periods evaluated for trastuzumab or ramucirumab in claims, CCQP or EHR data. Treatment augmentation changed with ramucirumab ( $p=0.03$, Table 2$)$, but not with trastuzumab $(p=0.58$, Table 1$)$.

As shown in Tables 1 and 2, the time from initial diagnosis to initiation of trastuzumab therapy was significantly different during the periods evaluated $(p=0.002)$ but was not different across time periods for ramucirumab $(\mathrm{p}=0.49)$. Tukey's HSD confirmed that for trastuzumab there were significant differences between the year 2010 and the years 2013-2017 in terms of the mean time from diagnosis to therapy initiation $(\mathrm{p}<0.05)$; for ramucirumab, the test found no significant differences in this metric across years.

As demonstrated in Figure 2, the trend to earlier lines of therapy was evident for trastuzumab for earlier years (20102013) versus after 2013 ( $\mathrm{p}=0.03$ for a comparison of trend lines from linear regression). Although less clear for 
Table 2 Ramucirumab Claims Cohort, Overall and by Index Half-Year

\begin{tabular}{|c|c|c|c|c|c|c|c|c|}
\hline \multicolumn{9}{|c|}{ Demographic and Clinical Characteristics } \\
\hline & Overall & $\begin{array}{l}\text { April - } \\
\text { December } 2014\end{array}$ & $\begin{array}{l}\text { January - } \\
\text { June } 2015\end{array}$ & $\begin{array}{l}\text { July - } \\
\text { December } 2015\end{array}$ & $\begin{array}{l}\text { January - } \\
\text { June } 2016\end{array}$ & $\begin{array}{l}\text { July - } \\
\text { December } 2016\end{array}$ & $\begin{array}{l}\text { January - } \\
\text { April } 2017\end{array}$ & p-value ${ }^{a}$ \\
\hline $\mathbf{N}$ & 287 & 38 & 50 & 41 & 71 & 47 & 40 & \\
\hline \multicolumn{9}{|l|}{ Age at index date, years } \\
\hline Mean (SD) & $60.0(11.1)$ & $58.5(8.7)$ & $59.0(12.3)$ & $58.6(12.0)$ & $59.7(9.7)$ & $61.3(11.6)$ & $63.2(12.1)$ & 0.33 \\
\hline \multicolumn{9}{|l|}{ Gender, $\mathbf{n}(\%)$} \\
\hline Female & $72(25.1)$ & $7(18.4)$ & II (22.0) & $10(24.4)$ & $15(21.1)$ & $12(25.5)$ & $17(42.5)$ & 0.15 \\
\hline Male & $215(74.9)$ & $31(81.6)$ & $39(78.0)$ & $31(75.6)$ & $56(78.9)$ & $35(74.5)$ & $23(57.5)$ & \\
\hline \multicolumn{8}{|l|}{ Health plan type, $n$ (\%) } & 0.01 \\
\hline HMO & $56(19.5)$ & $6(15.8)$ & $8(16.0)$ & II (26.8) & $10(14.1)$ & $4(8.5)$ & $17(42.5)$ & 0.001 \\
\hline PPO & $186(64.8)$ & $26(68.4)$ & $33(66.0)$ & $26(63.4)$ & $46(64.8)$ & $35(74.5)$ & $20(50.0)$ & 0.30 \\
\hline $\mathrm{CDHP}$ & $45(15.7)$ & $6(15.8)$ & $9(18.0)$ & $4(9.8)$ & $15(21.1)$ & $8(17.0)$ & $3(7.5)$ & 0.42 \\
\hline \multicolumn{9}{|c|}{ Non-commercial enrollment, $\mathbf{n}(\%)$} \\
\hline $\begin{array}{l}\text { Medicare Advantage, } \\
\text { Supplemental, or Part D }\end{array}$ & $52(18.1)$ & $7(18.4)$ & $6(12.0)$ & $7(17.1)$ & $9(12.7)$ & $10(21.3)$ & $13(32.5)$ & 0.13 \\
\hline \multicolumn{9}{|l|}{ Geographic region, n (\%) } \\
\hline Northeast & $56(19.5)$ & $6(15.8)$ & $13(26.0)$ & $12(29.3)$ & $13(18.3)$ & $5(10.6)$ & $7(17.5)$ & 0.25 \\
\hline Midwest & $60(20.9)$ & $8(21.1)$ & $5(10.0)$ & $6(14.6)$ & $18(25.4)$ & II (23.4) & $12(30.0)$ & 0.18 \\
\hline South & $80(27.9)$ & $12(31.6)$ & $14(28.0)$ & II (26.8) & $21(29.6)$ & $10(21.3)$ & $12(30.0)$ & 0.91 \\
\hline West & $91(31.7)$ & $12(31.6)$ & $18(36.0)$ & $12(29.3)$ & $19(26.8)$ & $21(44.7)$ & $9(22.5)$ & 0.26 \\
\hline \multicolumn{9}{|c|}{ Site of care at index infusion, $\mathbf{n}(\%)$} \\
\hline Physician office visit & $112(39.0)$ & $14(36.8)$ & II (22.0) & $13(31.7)$ & $29(40.8)$ & $25(53.2)$ & $20(50.0)$ & 0.02 \\
\hline Outpatient hospital & $170(59.2)$ & $22(57.9)$ & $38(76.0)$ & $27(65.9)$ & $41(57.7)$ & $22(46.8)$ & $20(50.0)$ & 0.05 \\
\hline Inpatient hospital/ER & I (0.3) & $0(0)$ & I (2.0) & $0(0)$ & $0(0)$ & $0(0)$ & $0(0)$ & 0.75 \\
\hline All other locations & $4(1.4)$ & $2(5.3)$ & $0(0)$ & I (2.4) & $\mathrm{I}(\mathrm{I} .4)$ & $0(0)$ & $0(0)$ & 0.26 \\
\hline \multicolumn{9}{|c|}{ Time from initial diagnosis to index infusion, days } \\
\hline Median (IQR) & $232.0(225.0)$ & $254.0(214.0)$ & $255.5(248.0)$ & $201.0(185.0)$ & $242.0(289.0)$ & $238.0(167.0)$ & $218.0(257.0)$ & \\
\hline Mean (SD) & $217.7(123.6)$ & $225.6(121.4)$ & $222.8(134.7)$ & $204.4(116.8)$ & $206.5(137.2)$ & $246.8(84.0)$ & $203.5(132.3)$ & 0.49 \\
\hline \multicolumn{9}{|c|}{ Follow-up time period, days } \\
\hline Median (IQR) & $160.0(174.0)$ & $114.0(123.0)$ & $251.5(225.0)$ & $128.0(152.0)$ & $193.0(156.0)$ & $141.0(203.0)$ & $135.0(72.5)$ & \\
\hline Mean (SD) & $203.7(161.6)$ & $182.6(205.5)$ & $280.4(179.8)$ & $193.3(196.5)$ & $222.9(144.1)$ & $175.4(117.3)$ & $137.8(59.8)$ & 0.001 \\
\hline \multicolumn{9}{|c|}{ Quan Charlson Comorbidity Items, n (\%) } \\
\hline Myocardial infarction & $7(2.4)$ & $2(5.3)$ & $2(4.0)$ & $0(0)$ & I (1.4) & $\mathrm{I}(2.1)$ & $\mathrm{I}(2.5)$ & 0.70 \\
\hline Congestive heart failure & $28(9.8)$ & $6(15.8)$ & $4(8.0)$ & $3(7.3)$ & $3(4.2)$ & $7(14.9)$ & $5(12.5)$ & 0.25 \\
\hline $\begin{array}{l}\text { Peripheral vascular } \\
\text { disease }\end{array}$ & $32(11.1)$ & $4(10.5)$ & $3(6.0)$ & $2(4.9)$ & $9(12.7)$ & $8(17.0)$ & $6(15.0)$ & 0.37 \\
\hline Cerebrovascular disease & $15(5.2)$ & $\mathrm{I}(2.6)$ & $4(8.0)$ & $2(4.9)$ & $3(4.2)$ & $2(4.3)$ & $3(7.5)$ & 0.87 \\
\hline Dementia & $0(0)$ & $0(0)$ & $0(0)$ & $0(0)$ & $0(0)$ & $0(0)$ & $0(0)$ & NE \\
\hline $\begin{array}{l}\text { Chronic pulmonary } \\
\text { disease }\end{array}$ & $50(17.4)$ & $7(18.4)$ & $6(12.0)$ & $10(24.4)$ & $12(16.9)$ & $8(17.0)$ & $7(17.5)$ & 0.78 \\
\hline $\begin{array}{l}\text { Connective tissue/ } \\
\text { rheumatic disease }\end{array}$ & $2(0.7)$ & I (2.6) & $0(0)$ & $0(0)$ & $0(0)$ & I (2.I) & $0(0)$ & 0.27 \\
\hline
\end{tabular}

(Continued) 
Table 2 (Continued).

\begin{tabular}{|c|c|c|c|c|c|c|c|c|}
\hline \multicolumn{9}{|c|}{ Demographic and Clinical Characteristics } \\
\hline & Overall & $\begin{array}{l}\text { April - } \\
\text { December } 2014\end{array}$ & $\begin{array}{l}\text { January - } \\
\text { June } 2015\end{array}$ & $\begin{array}{l}\text { July - } \\
\text { December } 2015\end{array}$ & $\begin{array}{l}\text { January - } \\
\text { June } 2016\end{array}$ & $\begin{array}{l}\text { July - } \\
\text { December } 2016\end{array}$ & $\begin{array}{l}\text { January - } \\
\text { April } 2017\end{array}$ & p-value ${ }^{a}$ \\
\hline $\mathbf{N}$ & 287 & 38 & 50 & 41 & 71 & 47 & 40 & \\
\hline $\begin{array}{l}\text { Peptic ulcer disease } \\
\text { Liver disease }\end{array}$ & $23(8.0)$ & $2(5.3)$ & $6(12.0)$ & $4(9.8)$ & $3(4.2)$ & $2(4.3)$ & $6(15.0)$ & 0.26 \\
\hline Mild & $93(32.4)$ & $15(39.5)$ & $20(40.0)$ & II (26.8) & $18(25.4)$ & $16(34.0)$ & $13(32.5)$ & 0.50 \\
\hline Moderate or severe & $3(1.0)$ & $0(0)$ & I (2.0) & I (2.4) & $0(0)$ & $\mathrm{I}(2.1)$ & $0(0)$ & 0.55 \\
\hline Paraplegia and hemiplegia & $\mathrm{I}(0.3)$ & $0(0)$ & I (2.0) & $0(0)$ & $0(0)$ & $0(0)$ & $0(0)$ & 0.75 \\
\hline $\begin{array}{l}\text { Renal disease } \\
\text { Diabetes }\end{array}$ & $18(6.3)$ & $3(7.9)$ & $2(4.0)$ & $2(4.9)$ & $2(2.8)$ & $4(8.5)$ & $5(12.5)$ & 0.39 \\
\hline $\begin{array}{l}\text { Diabetes without chronic } \\
\text { complications }\end{array}$ & $49(17.1)$ & II (28.9) & $9(18.0)$ & $8(19.5)$ & $8(11.3)$ & $6(12.8)$ & $7(17.5)$ & 0.28 \\
\hline $\begin{array}{l}\text { Diabetes with chronic } \\
\text { complications }\end{array}$ & II (3.8) & $2(5.3)$ & $3(6.0)$ & I (2.4) & I ( $(1.4)$ & $I(2.1)$ & $3(7.5)$ & 0.51 \\
\hline Cancer & $200(69.7)$ & $31(81.6)$ & $28(56.0)$ & $28(68.3)$ & $54(76.1)$ & $37(78.7)$ & $22(55.0)$ & 0.01 \\
\hline Metastatic carcinoma & $242(84.3)$ & $36(94.7)$ & $39(78.0)$ & $35(85.4)$ & $58(81.7)$ & $42(89.4)$ & $32(80.0)$ & 0.26 \\
\hline AIDS/HIV & $0(0)$ & $0(0)$ & $0(0)$ & $0(0)$ & $0(0)$ & $0(0)$ & $0(0)$ & NE \\
\hline \multicolumn{9}{|c|}{ Quan Charlson Comorbidity Index } \\
\hline Mean (SD) & $7.7(3.1)$ & $8.9(2.6)$ & $7.2(3.2)$ & $7.7(3.1)$ & $7.3(3.0)$ & $8.3(3.3)$ & $7.5(3.2)$ & 0.08 \\
\hline \multicolumn{9}{|c|}{ Procedures during baseline period (n, \%) } \\
\hline $\begin{array}{l}\text { Surgery (endoscopic } \\
\text { mucosal resection; } \\
\text { gastrectomy) }\end{array}$ & $29(10.1)$ & I (2.6) & $5(10.0)$ & $2(4.9)$ & $8(11.3)$ & $7(14.9)$ & $6(15.0)$ & 0.29 \\
\hline Radiation & $66(23.0)$ & $10(26.3)$ & $9(18.0)$ & $8(19.5)$ & $16(22.5)$ & $16(34.0)$ & $7(17.5)$ & 0.40 \\
\hline HER2 testing & $96(33.4)$ & $12(31.6)$ & $18(36.0)$ & $13(31.7)$ & $17(23.9)$ & $22(46.8)$ & $14(35.0)$ & 0.22 \\
\hline \multicolumn{9}{|l|}{ Treatment Patterns } \\
\hline \multicolumn{9}{|c|}{ Line of therapy in which ramucirumab was initiated, $n(\%)$} \\
\hline First line & $46(16.0)$ & I (2.6) & $10(20.0)$ & $5(12.2)$ & $17(23.9)$ & $3(6.4)$ & $10(25.0)$ & 0.01 \\
\hline Second line & $125(43.6)$ & $10(26.3)$ & $25(50.0)$ & $21(51.2)$ & $28(39.4)$ & $26(55.3)$ & $15(37.5)$ & 0.07 \\
\hline Third line & $73(25.4)$ & II (28.9) & $10(20.0)$ & $14(34.1)$ & $16(22.5)$ & $12(25.5)$ & $10(25.0)$ & 0.70 \\
\hline Fourth line & $26(9.1)$ & II (28.9) & $3(6.0)$ & I (2.4) & $6(8.5)$ & $2(4.3)$ & $3(7.5)$ & 0.003 \\
\hline Fifth line & $10(3.5)$ & $3(7.9)$ & $I(2.0)$ & $0(0)$ & $3(4.2)$ & $2(4.3)$ & I (2.5) & 0.55 \\
\hline Sixth line & $4(1.4)$ & I (2.6) & I (2.0) & $0(0)$ & I (I.4) & I (2.1) & $0(0)$ & 0.95 \\
\hline Seventh line & $3(1.0)$ & I (2.6) & $0(0)$ & $0(0)$ & $0(0)$ & I (2.1) & I (2.5) & 0.31 \\
\hline \multicolumn{8}{|c|}{ Regimen in which ramucirumab was initiated ${ }^{c}, n(\%)$} & NE \\
\hline Ramucirumab only & $207(72.1)$ & $8(21.1)$ & $34(68.0)$ & $23(56.1)$ & $63(88.7)$ & $42(89.4)$ & $37(92.5)$ & \\
\hline $\begin{array}{l}\text { Ramucirumab } \\
\text { +unclassified }\end{array}$ & $24(8.4)$ & $8(21.1)$ & $8(16.0)$ & $8(19.5)$ & $0(0)$ & $0(0)$ & $0(0)$ & \\
\hline Ramucirumab+paclitaxel & $7(2.4)$ & $7(18.4)$ & $0(0)$ & $0(0)$ & $0(0)$ & $0(0)$ & $0(0)$ & \\
\hline $\begin{array}{l}\text { Ramucirumab } \\
\text { +unclassified biologics }\end{array}$ & $7(2.4)$ & $0(0)$ & $2(4.0)$ & $4(9.8)$ & I ( 1.4$)$ & $0(0)$ & $0(0)$ & \\
\hline $\begin{array}{l}\text { Ramucirumab } \\
\text { +unclassified+paclitaxel }\end{array}$ & $7(2.4)$ & $7(18.4)$ & $0(0)$ & $0(0)$ & $0(0)$ & $0(0)$ & $0(0)$ & \\
\hline Ramucirumab+FOLFIRI & $6(2.1)$ & $0(0)$ & $2(4.0)$ & I (2.4) & I (I.4) & I (2.1) & I (2.5) & \\
\hline $\begin{array}{l}\text { Ramucirumab } \\
\text { +trastuzumab }\end{array}$ & $4(1.4)$ & $0(0)$ & $0(0)$ & $2(4.9)$ & $0(0)$ & $2(4.3)$ & $0(0)$ & \\
\hline
\end{tabular}


Table 2 (Continued).

\begin{tabular}{|c|c|c|c|c|c|c|c|c|}
\hline \multicolumn{9}{|c|}{ Demographic and Clinical Characteristics } \\
\hline & Overall & $\begin{array}{l}\text { April - } \\
\text { December } 2014\end{array}$ & $\begin{array}{l}\text { January - } \\
\text { June } 2015\end{array}$ & $\begin{array}{l}\text { July - } \\
\text { December } 2015\end{array}$ & $\begin{array}{l}\text { January - } \\
\text { June } 2016\end{array}$ & $\begin{array}{l}\text { July - } \\
\text { December } 2016\end{array}$ & $\begin{array}{l}\text { January - } \\
\text { April } 2017\end{array}$ & p-value ${ }^{a}$ \\
\hline $\mathbf{N}$ & 287 & 38 & 50 & 41 & 71 & 47 & 40 & \\
\hline $\begin{array}{l}\text { Ramucirumab } \\
\text { +fluoropyrimidine }\end{array}$ & $3(1.0)$ & $0(0)$ & I (2.0) & $0(0)$ & I (I.4) & I (2.1) & $0(0)$ & \\
\hline Ramucirumab+irinotecan & $3(1.0)$ & I (2.6) & $0(0)$ & I (2.4) & $0(0)$ & $0(0)$ & $\mathrm{I}(2.5)$ & \\
\hline Unclassified+paclitaxel & $3(1.0)$ & $2(5.3)$ & I (2.0) & $0(0)$ & $0(0)$ & $0(0)$ & $0(0)$ & \\
\hline $\begin{array}{l}\text { Augmentation with } \\
\text { ramucirumab }^{\mathrm{d}}, \mathrm{n}(\%)\end{array}$ & $10(3.5)$ & $5(13.2)$ & $2(4.0)$ & $0(0)$ & $2(2.8)$ & I (2.1) & $0(0)$ & 0.03 \\
\hline \multicolumn{9}{|c|}{ Duration of ramucirumab therapy, days } \\
\hline RMST (SD) & $122.0(7.7)$ & $86.0(15.5)$ & $147.0(18.1)$ & $90.1(12.6)$ & $117.0(14.1)$ & $124.3(17.7)$ & $108.8(10.8)$ & 0.13 \\
\hline
\end{tabular}

Notes: ${ }^{\mathrm{a}} \mathrm{p}$-values were based on F-test for continuous variables and Chi-squared/Fisher-exact test for categorical variables. Duration of therapy was compared using a Logrank test. ${ }^{b}$ Gastric cancer (International Classification of Disease, ICD-9-CM, clinical modification, codes: I5I; ICD-I0-CM codes: CI6) were excluded in identifying cancer conditions. ' Limited to regimens with $\mathrm{n}>\mathrm{I}$. ${ }^{\mathrm{d}}$ Ramucirumab was added to the chemotherapy regimen. Bolded text represents column headers and $\mathrm{p}$-values $<0.05$.

Abbreviations: HMO, health maintenance organization; PPO, preferred provider organization; CDHP, consumer-directed health plan; AIDS/HIV, acquired immune deficiency syndrome/human immunodeficiency virus; HER2, human epidermal growth factor receptor 2; ER, emergency room; NE, not estimable; SD, standard deviation; IQR, interquartile range; RMST, restricted mean survival time; FOLFIRI, fluoropyrimidine + irinotecan.

ramucirumab, there is a jagged trend toward increased use as first-line therapy in the claims data $(\mathrm{p}=0.010)$. In both the trastuzumab and ramucirumab claims cohorts there is a visual difference in the first year of approval measured versus the later years, which is not seen in the CCQP or EHR cohort with ramucirumab. Both of those datasets, on the other hand, suggest a decreased use in third line or higher, and increased use in second-line therapy.

\section{Discussion and Conclusions}

Observational studies of these agents for gastric or GEJ cancer have generally included samples of very few patients $^{11-14}$ as this is a rare tumor type in the US; both agents included in this study met the FDA requirements for orphan drug status. ${ }^{15}$ This study of 1700 patients suggests that trastuzumab was initially administered in later lines of therapy before migrating to earlier lines over time. This was less apparent for ramucirumab, which was approved as post-progression therapy. This could in part be due to an initially large number of eligible patients in advanced stages of therapy who had not had the opportunity to receive these agents in earlier lines due to the timing of the FDA approval. Fluctuating treatment guidelines may also play a role. As newly diagnosed patients enter the health care system, providers would have more time to plan the potential options for therapy in the future that included this new drug, whereas patients who had already received prior therapy at the time of drug approval may have received the drug at a later change in line of therapy. From this study, it appears that the process of incorporating trastuzumab into clinical practice took approximately 1 year as noted by the changes in patterns from 2010 to 2011; however, data from the year of approval through 2013 demonstrated many differences as well, suggesting that even after one-year modifications to treatment patterns can occur. On the other hand, the use of ramucirumab, which was only approved for use after disease progression, was relatively more consistent after approval, suggesting that the pattern of incorporating a new drug into clinical practice could vary considerably.

Delays in the introduction of trastuzumab into the firstline setting in appropriate patients could be explained by a number of factors including increased awareness of the HER2-positive subset of gastric cancer patients, implementation of standardized testing, and increased experience with the use of trastuzumab. It is also interesting to note there was a concurrent evolution in the National Comprehensive Cancer Network (NCCN) clinical practice guidelines that may have influenced treatment patterns: In 2010, trastuzumab in combination with chemotherapy was included in the guidelines for HER2-positive metastatic or locally advanced gastric cancer, which differed from the labeled indication for first-line use. ${ }^{16}$ Changes in the NCCN and other clinical guidelines may have impacted observed treatment patterns for trastuzumab and ramucirumab. 
Table 3 Ramucirumab Cancer Care Quality Program (CCQP) Cohort, Overall and by Index Year

\begin{tabular}{|c|c|c|c|c|c|c|c|}
\hline \multicolumn{8}{|c|}{ Demographic and Clinical Characteristics } \\
\hline Variable Name & Overall & 2014 & 2015 & 2016 & 2017 & 2018 & p-value ${ }^{a}$ \\
\hline $\mathbf{N}$ & 244 & 15 & 59 & 66 & 78 & 26 & \\
\hline \multicolumn{8}{|l|}{ Age at index date, years } \\
\hline Mean (SD) & $56.5(9.92)$ & $55.1(8.48)$ & $57.5(10.51)$ & $55.5(9.20)$ & $56.4(11.05)$ & $58.1(7.34)$ & 0.69 \\
\hline \multicolumn{8}{|l|}{ Gender, n (\%) } \\
\hline $\begin{array}{l}\text { Female } \\
\text { Male }\end{array}$ & $\begin{array}{l}80(32.8) \\
164(67.2)\end{array}$ & $\begin{array}{l}4(26.7) \\
\text { II (73.3) }\end{array}$ & $\begin{array}{l}22(37.3) \\
37(62.7)\end{array}$ & $\begin{array}{l}22(33.3) \\
44(66.7)\end{array}$ & $\begin{array}{l}27(34.6) \\
51(65.4)\end{array}$ & $\begin{array}{l}5(19.2) \\
21(80.8)\end{array}$ & 0.76 \\
\hline \multicolumn{8}{|l|}{ Health plan type, $n(\%)$} \\
\hline $\begin{array}{l}\text { HMO } \\
\text { PPO } \\
\text { CDHP }\end{array}$ & $\begin{array}{l}60(24.6) \\
149(61.1) \\
35(14.3)\end{array}$ & $\begin{array}{l}2(13.3) \\
11(73.3) \\
2(13.3)\end{array}$ & $\begin{array}{l}15(25.4) \\
37(62.7) \\
7(11.9)\end{array}$ & $\begin{array}{l}12(18.2) \\
46(69.7) \\
8(12.1)\end{array}$ & $\begin{array}{l}28(35.9) \\
40(51.3) \\
10(12.8)\end{array}$ & $\begin{array}{l}3(11.5) \\
15(57.7) \\
8(30.8)\end{array}$ & $\begin{array}{l}0.04 \\
0.18 \\
0.17\end{array}$ \\
\hline \multicolumn{8}{|l|}{ Non-commercial enrollment, $n$ (\%) } \\
\hline Medicare Advantage & $17(7.0)$ & I (6.7) & $6(10.2)$ & $3(4.5)$ & $6(7.7)$ & I (3.8) & 0.76 \\
\hline \multicolumn{8}{|l|}{ Geographic region, $\mathbf{n}(\%)$} \\
\hline $\begin{array}{l}\text { Northeast } \\
\text { Midwest } \\
\text { South } \\
\text { West }\end{array}$ & $\begin{array}{l}49(20.1) \\
49(20.1) \\
76(31.1) \\
70(28.7)\end{array}$ & $\begin{array}{l}0(0) \\
5(33.3) \\
4(26.7) \\
6(40.0)\end{array}$ & $\begin{array}{l}13(22.0) \\
13(22.0) \\
11(18.6) \\
22(37.3)\end{array}$ & $\begin{array}{l}10(15.2) \\
12(18.2) \\
20(30.3) \\
24(36.4)\end{array}$ & $\begin{array}{l}21(26.9) \\
16(20.5) \\
30(38.5) \\
11(14.1)\end{array}$ & $\begin{array}{l}5(19.2) \\
3(11.5) \\
11(42.3) \\
7(26.9)\end{array}$ & $\begin{array}{l}0.13 \\
0.54 \\
0.10 \\
\mathbf{0 . 0 1}\end{array}$ \\
\hline \multicolumn{8}{|l|}{ ECOG performance status, n (\%) } \\
\hline $\begin{array}{l}0 \\
1 \\
2 \\
3 \\
4\end{array}$ & $\begin{array}{l}54(22.1) \\
137(56.1) \\
25(10.2) \\
I(0.4) \\
I(0.4)\end{array}$ & $\begin{array}{l}3(20.0) \\
12(80.0) \\
0(0) \\
0(0) \\
0(0)\end{array}$ & $\begin{array}{l}\text { II (18.6) } \\
38(64.4) \\
5(8.5) \\
0(0) \\
0(0)\end{array}$ & $\begin{array}{l}16(24.2) \\
37(56.1) \\
5(7.6) \\
0(0) \\
1(1.5)\end{array}$ & $\begin{array}{l}18(23.1) \\
37(47.4) \\
10(12.8) \\
1(1.3) \\
0(0)\end{array}$ & $\begin{array}{l}6(23.1) \\
13(50.0) \\
5(19.2) \\
0(0) \\
0(0)\end{array}$ & $\begin{array}{l}0.95 \\
0.10 \\
0.26 \\
1.00 \\
0.68\end{array}$ \\
\hline \multicolumn{8}{|l|}{ HER2 status, n (\%) } \\
\hline $\begin{array}{l}\text { Negative } \\
\text { Positive } \\
\text { Conflicting }\end{array}$ & $\begin{array}{l}|4|(75.8) \\
3 \mid(16.7) \\
1 \mid(5.9)\end{array}$ & $\begin{array}{l}5(50.0) \\
0(0) \\
3(30.0)\end{array}$ & $\begin{array}{l}32(76.2) \\
8(19.0) \\
1(2.4)\end{array}$ & $\begin{array}{l}39(79.6) \\
8(16.3) \\
2(4.1)\end{array}$ & $\begin{array}{l}49(75.4) \\
12(18.5) \\
4(6.2)\end{array}$ & $\begin{array}{l}16(80.0) \\
3(15.0) \\
1(5.0)\end{array}$ & $\begin{array}{l}0.38 \\
0.67 \\
0.07\end{array}$ \\
\hline \multicolumn{8}{|l|}{ Treatment patterns } \\
\hline \multicolumn{8}{|c|}{ Line of therapy in which ramucirumab was initiated, $n$ (\%) } \\
\hline $\begin{array}{l}\text { First line } \\
\text { Second line } \\
\text { Third line } \\
\text { Fourth line or higher }\end{array}$ & $\begin{array}{l}12(4.9) \\
149(61.1) \\
54(22.1) \\
29(11.9)\end{array}$ & $\begin{array}{l}0(0) \\
7(46.7) \\
5(33.3) \\
3(20.0)\end{array}$ & $\begin{array}{l}4(6.8) \\
33(55.9) \\
15(25.4) \\
7(11.9)\end{array}$ & $\begin{array}{l}2(3.0) \\
40(60.6) \\
14(21.2) \\
10(15.2)\end{array}$ & $\begin{array}{l}4(5.1) \\
51(65.4) \\
16(20.5) \\
7(9.0)\end{array}$ & $\begin{array}{l}2(7.7) \\
18(69.2) \\
4(15.4) \\
2(7.7)\end{array}$ & $\begin{array}{l}0.72 \\
0.51 \\
0.68 \\
0.61\end{array}$ \\
\hline \multicolumn{8}{|c|}{ Regimen in which ramucirumab was initiated, $\mathbf{n}(\%)$} \\
\hline $\begin{array}{l}\text { Ramucirumab+paclitaxel } \\
\text { Ramucirumab } \\
\text { Ramucirumab+FOLFIRI } \\
\text { Ramucirumab+docetaxel } \\
\text { Ramucirumab+carboplatin+paclitaxel } \\
\text { Ramucirumab+paclitaxel+trastuzumab } \\
\text { Ramucirumab+denosumab+paclitaxel }\end{array}$ & $\begin{array}{l}177(72.5) \\
43(17.6) \\
7(2.9) \\
4(1.6) \\
3(1.2) \\
3(1.2) \\
1(0.4)\end{array}$ & $\begin{array}{l}\text { II (73.3) } \\
4(26.7) \\
0(0) \\
0(0) \\
0(0) \\
0(0) \\
0(0)\end{array}$ & $\begin{array}{l}40(67.8) \\
9(15.3) \\
1(1.7) \\
3(5.1) \\
3(5.1) \\
0(0) \\
0(0)\end{array}$ & $\begin{array}{l}48(72.7) \\
13(19.7) \\
2(3.0) \\
0(0) \\
0(0) \\
2(3.0) \\
0(0)\end{array}$ & $\begin{array}{l}60(76.9) \\
13(16.7) \\
1(1.3) \\
0(0) \\
0(0) \\
1(1.3) \\
1(1.3)\end{array}$ & $\begin{array}{l}18(69.2) \\
4(15.4) \\
3(11.5) \\
1(3.8) \\
0(0) \\
0(0) \\
0(0)\end{array}$ & $\begin{array}{l}0.82 \\
0.84 \\
0.14 \\
0.07 \\
0.11 \\
0.72 \\
1.00\end{array}$ \\
\hline
\end{tabular}


Table 3 (Continued).

\begin{tabular}{|c|c|c|c|c|c|c|c|}
\hline \multicolumn{8}{|c|}{ Demographic and Clinical Characteristics } \\
\hline Variable Name & Overall & 2014 & 2015 & 2016 & 2017 & 2018 & $p$-value ${ }^{a}$ \\
\hline $\mathbf{N}$ & 244 & 15 & 59 & 66 & 78 & 26 & \\
\hline Ramucirumab+docetaxel+irinotecan & I (0.4) & $0(0)$ & $0(0)$ & $0(0)$ & $\mathrm{I}(1.3)$ & $0(0)$ & 1.00 \\
\hline Ramucirumab+FOLFOX & $\mathrm{I}(0.4)$ & $0(0)$ & I (I.7) & $0(0)$ & $0(0)$ & $0(0)$ & 0.41 \\
\hline $\begin{array}{l}\text { Ramucirumab+irinotecan+oxaliplatin+ } \\
\text { trastuzumab }\end{array}$ & I (0.4) & $0(0)$ & $\mathrm{I}(\mathrm{I} .7)$ & $0(0)$ & $0(0)$ & $0(0)$ & 0.41 \\
\hline Ramucirumab+irinotecan & I (0.4) & $0(0)$ & $0(0)$ & $\mathrm{I}(\mathrm{l} .5)$ & $0(0)$ & $0(0)$ & 0.68 \\
\hline Ramucirumab+nivolumab & I (0.4) & $0(0)$ & I (I.7) & $0(0)$ & $0(0)$ & $0(0)$ & 0.41 \\
\hline Ramucirumab+pembrolizumab & I $(0.4)$ & $0(0)$ & $0(0)$ & $0(0)$ & $\mathrm{I}(\mathrm{I} .3)$ & $0(0)$ & 1.00 \\
\hline \multicolumn{8}{|c|}{ Time from first to last pre-authorization for ramucirumab therapy, days ${ }^{b}$} \\
\hline $\mathrm{N}(\%$ of total) & $55(22.5)$ & $3(20.0)$ & II (18.6) & $19(28.8)$ & $21(26.9)$ & I (3.8) & \\
\hline Median (IQR) & $164.0(135.0)$ & $110.0(68.0)$ & $189.0(50.0)$ & $169.0(125.0)$ & $116.0(137.0)$ & $84.0(0)$ & \\
\hline Mean (SD) & $157.8(118.34)$ & $96.0(36.10)$ & $239.0(161.74)$ & $172.2(90.18)$ & $114.7(103.25)$ & $84.0()$. & 0.04 \\
\hline
\end{tabular}

Notes: ${ }^{\mathrm{a}} \mathrm{p}$-values were based on F-test for continuous variables and Chi-squared/Fisher's exact test for categorical variables. Duration of therapy was compared using a Logrank test. 'bimited to patients with $\geq 2$ preauthorizations. Bolded text represents column headers and $\mathrm{p}$-values $<0.05$.

Abbreviations: HMO, health maintenance organization; PPO, preferred provider organization; CCQP, cancer care quality program; CDHP, consumer-directed health plan; ECOG, Eastern Cooperative Oncology Group; HER2, human epidermal growth factor receptor 2; SD, standard deviation; IQR, interquartile range; FOLFIRI, fluoropyrimidine + irinotecan; FOLFOX, fluoropyrimidine + oxaliplatin.

This study was focused on gastric cancer, where few evidence-based treatment options are available. It is unknown if the observed changes in treatment patterns would hold true in another disease, such as breast or lung cancer, where multiple agents are FDA-approved and available for use. While claims and EHR data are valuable for the efficient and effective examination of healthcare outcomes, treatment patterns, resource utilization and costs, they are collected for the purpose of payment and patient care and not specifically for research. While use of these real-world datasets is a strength of the current study since the research question sought to understand the use of a new agent using big data, there are inherent limitations in claims and EHR data. First, there is a risk of incorrect cohort identification, in that the presence of a diagnosis code does not guarantee positive presence of a disease since the diagnosis code may be incorrectly coded or included as a rule-out criterion. We tried to limit this bias in the claims data by requiring at least two claims for gastric cancer on distinct dates. Next, results from the HIRD claims may not be generalizable to other data sources, such as EHR data, which are collected for different purposes. It is important to note that data from the final year of analysis were limited (full-year data were not available in 2018), affecting comparability to the other time periods. Gastric cancer is a relatively rare disease in the US, with only approximately 24,000 cases diagnosed per year, ${ }^{17}$ and trastuzumab is limited to HER2overexpressing cancers (limited to approximately $10-20 \%$ of all gastric cancers). ${ }^{18,19}$ Therefore, the sample size for the evaluation of trastuzumab as a new drug in gastric cancer is limited to a relatively small population. While the study sample used is small when compared to more common cancers (eg, breast cancer), it represents one of the largest studies to date of trastuzumab use in gastric cancer in a real-world setting (few observational studies have identified $>100$ trastuzumab patients). ${ }^{11-13}$ Finally, as with all claims-based analyses, the study results from the claims cohorts may not be fully generalizable to a larger population because patients who have commercial health insurance may have different characteristics from those without health insurance. Additionally, while there is likely little to no patient overlap between those patients in the claims and CCQP versus those entered in the EHR data source based on the regions and centers contributing data, minimizing the risk of patient duplication, this could not be fully evaluated in a de-identified dataset. Patients in the CCQP overlap strongly with those in claims, contributing additional consistency in these sources that may not be observed in completely independent data sources.

The CCQP and EHR data presented a different practice pattern (eg regimens and drug combinations were different from those observed the claims data), but the trends over time were consistent across these two ramucirumab cohorts. 


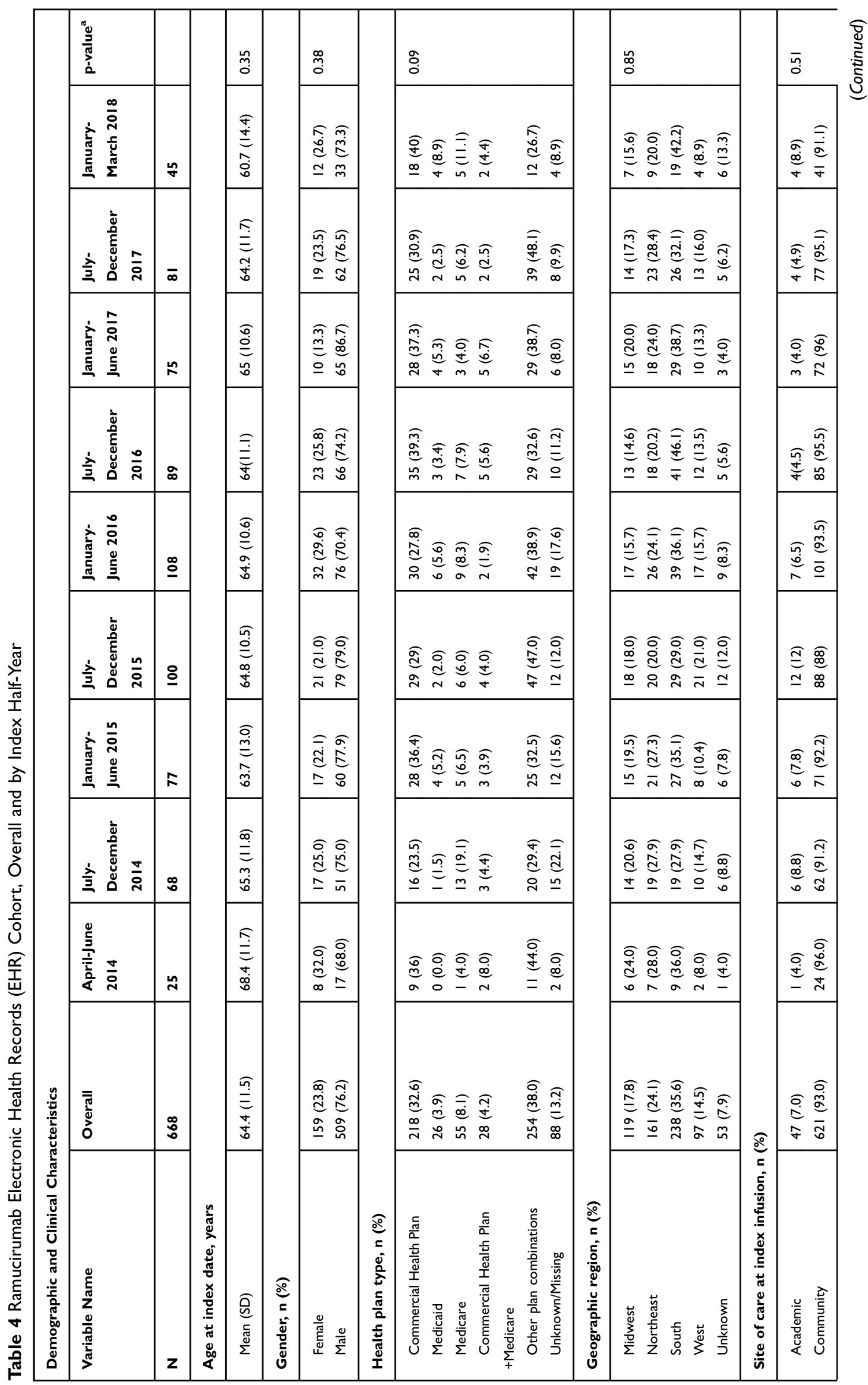




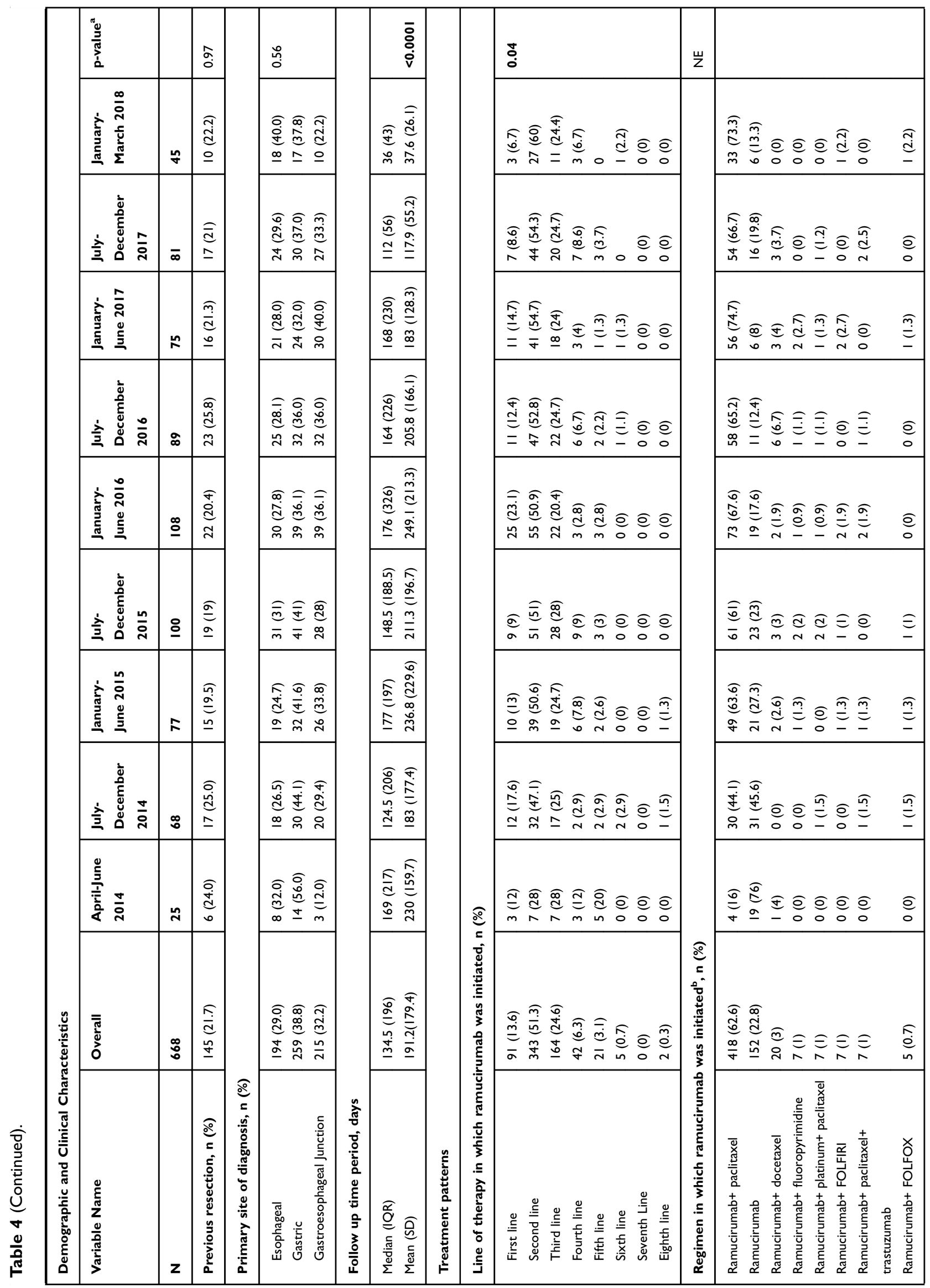




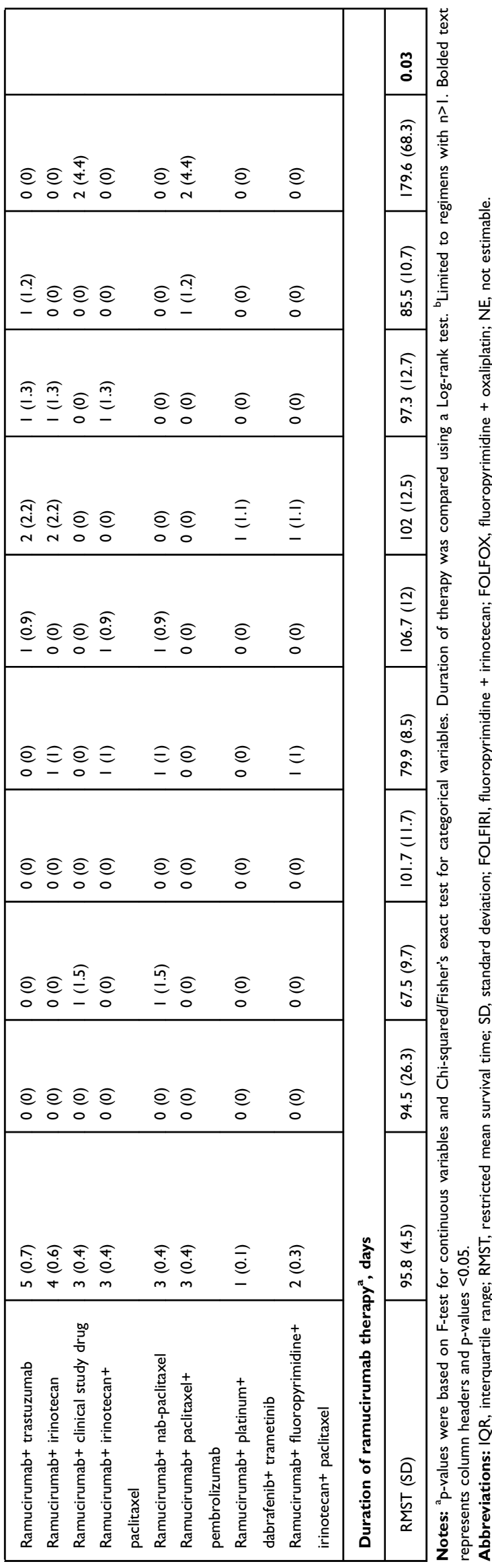

Differences in regimens with what was observed in the claims cohort could in part be due to the inability to fully capture the use of ramucirumab in claims prior to the release of the J-code, or due to coding entry practices. As claims data rely on coding to identify utilization, the coding strategies to observe a newly approved agent may fail to observe utilizations that are recorded by a generic coding system and may underestimate utilization during the time until final codes are issued. Additionally, the use of nonspecific drug codes in claims led to high rates of 'unclassified' drug use. These could be any number of agents and could have artificially consolidated disparate treatments in the claims data. Therefore, claims data alone should be used with caution within the immediate post-approval period. This uncertainty did not exist for trastuzumab, as it had previously been approved in other cancers and the codes were established at the time of its approval in gastric cancer. As actual drug names are entered into the CCQP and EHR systems, there are no unclassified drugs noted in these databases. The role of nonspecific coding should be explored in future studies to understand if there are further limitations in the use and interpretation of claims data. Further, the variables captured in EHR and claims differ, and few factors beyond basic demographics could be evaluated across both ramucirumab cohorts. Despite the observed differences in utilization, each ramucirumab cohort demonstrated a similar trend in evaluated clinical and demographic variables over time.

Based on this study, it is recommended that research evaluating treatment patterns or comparative effectiveness should use data shortly after post-approval with caution, particularly in the case of a drug with a first approval and using claims data alone. Treatment guidelines, such as NCCN in the US, should also be evaluated prior to the conduct of research. There is the possibility that fluctuating guidelines could alter use patterns and therefore influence the composition and outcomes of the cohort receiving a drug over time. This work also suggests that study results using either EHR or claims data for comparative effectiveness research may suffer bias based on differential treatment patterns. For example, if patients disproportionately receive treatment in later lines of therapy during the initial post-approval phase in one comparison cohort but not another, the benefit of a drug may be underestimated (patients whose disease has progressed several times tend to have reduced efficacy outcomes). ${ }^{20}$ To help reduce this bias, study designs should be augmented by sensitivity analyses that restrict the population under investigation 
A
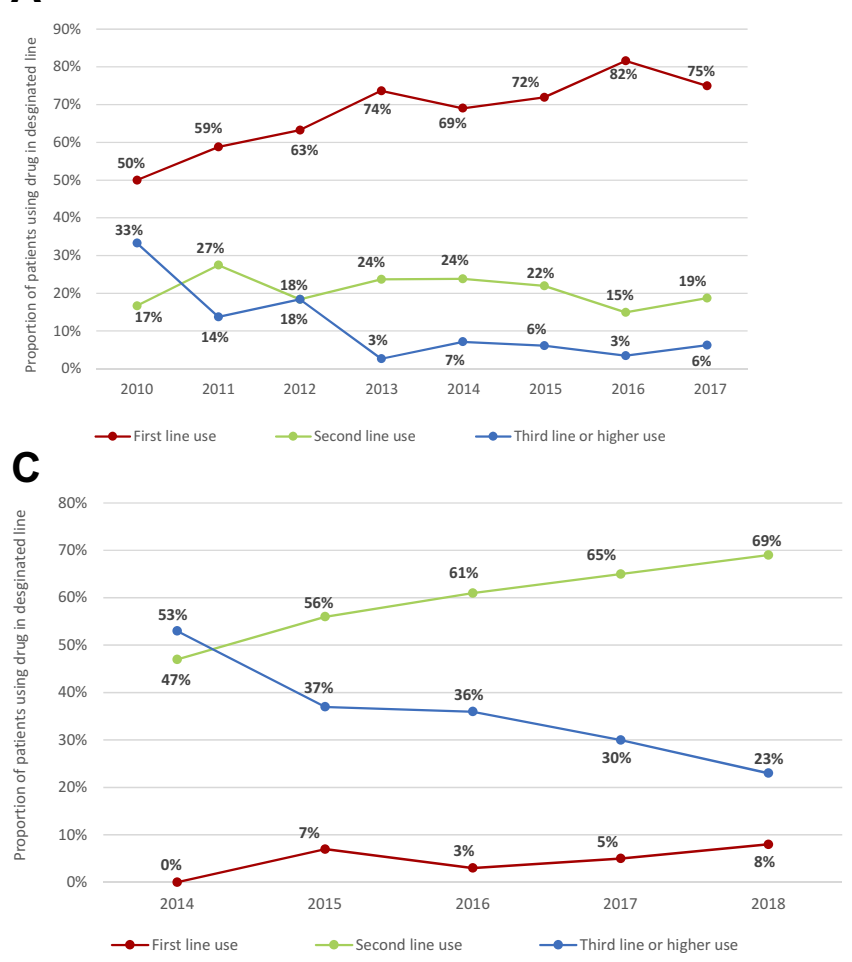

B

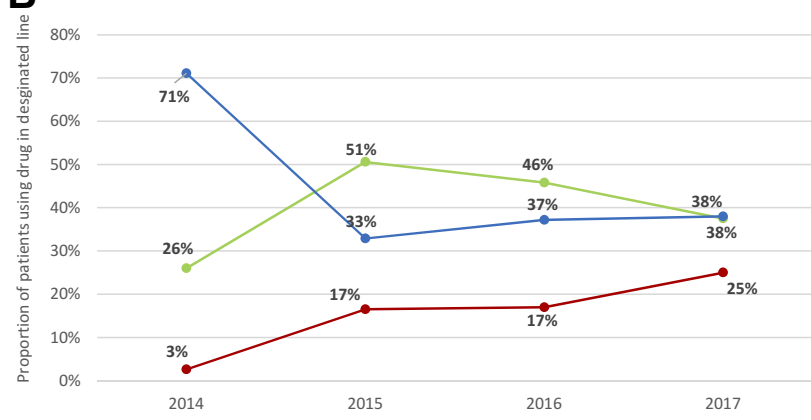

D

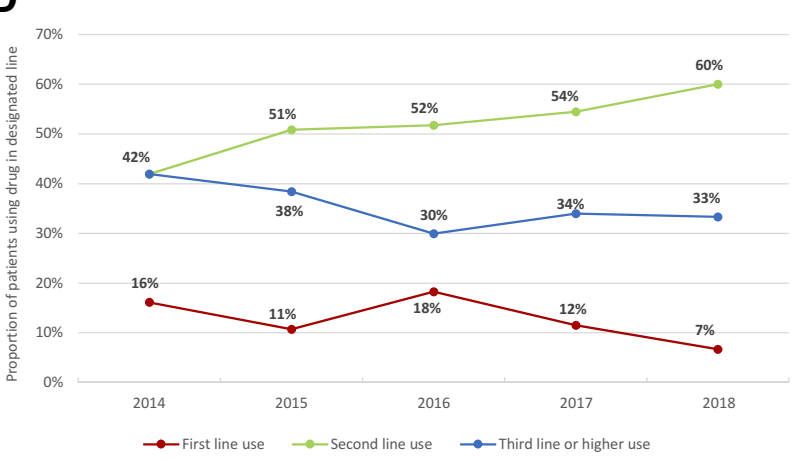

Figure 2 Trends in relative percent utilization by line of therapy over time: (A) Trastuzumab claims cohort; (B) Ramucirumab claims cohort; (C) Ramucirumab cancer care quality program cohort; (D) Ramucirumab electronic health records cohort.

to those with indications in accordance with treatment guidelines, drug labels, and/or by stratifying the population along key disease severity markers. This concurs with best practice guidelines for comparative effectiveness studies, where all characteristics of patients in the various study arms should be well-balanced prior to outcomes assessment. $^{21}$ As government and commercial payers are increasingly using administrative claims and other data to get early 'real world' signals of comparative effectiveness and benefit-risk trade-offs, the potential for bias associated with analyses of introductory clinical use of new drugs and the need for robust research designs should be acknowledged.

\section{Ethics and Consent Statement}

Flatiron EHR is a de-identified dataset, which is not considered human subjects research. Similarly, HealthCore data are only accessed in the form of a limited data set for which a data use agreement was in place with the covered entities in compliance with the Health Insurance Portability and Accountability (HIPAA) Privacy Rule.

\section{Data Sharing Statement}

Data for this study were used under contract with HealthCore and Flatiron Health, respectively, and are not available for distribution.

\section{Funding}

The conduct of this research was supported by funds to HealthCore, Inc. from Eli Lilly and Company. This research was a collaborative project between scientists at HealthCore, Inc, and Eli Lilly and Company; investigators from both organizations were involved in the project from conceptualization through submission for publication.

\section{Disclosure}

LMH, AML, XIL, ZLC, LB and WS are employees of Eli Lilly and Company. MG is an employee of HealthCore, Inc., an independent research organization that received funding from Eli Lilly and Company for the conduct of the study. LW was an employee of HealthCore, Inc. when the study was conducted. The authors report no other conflicts of interest in this work. 


\section{References}

1. Campbell RR, Spehar AM, French DD. Proactive postmarketing surveillance: overview and lessons learned from medication safety research in the veterans health administration. In: Henriksen K, Battles JB, Keyes MA, Grady ML, editors.Advances in Patient Safety: New Directions and Alternative Approaches (Vol. 1: Assessment).Rockville, MD: Agency for Healthcare Research and Quality;2008.

2. Woosley RL, Rice G. A new system for moving drugs to market. Issues Sci Technol. 2005;21:2.

3. Unger JM, Cook E, Tai E, Bleyer A. The role of clinical trial participation in cancer research: barriers, evidence, and strategies. Am Soc Clin Oncol. 2016;35:185-198.

4. Armstrong K. Methods in comparative effectiveness research. J clin oncol. 2012;30(34):4208-4214. doi:10.1200/JCO.2012.42.2659

5. Albert RK. "Lies, damned lies ..." and observational studies in comparative effectiveness research. Am J Respir Crit Care Med. 2013;187(11):1173-1177. doi:10.1164/rccm.201212-2187OE

6. Berger ML, Mamdani M, Atkins D, Johnson ML. Good research practices for comparative effectiveness research: defining, reporting and interpreting nonrandomized studies of treatment effects using secondary data sources: the ISPOR good research practices for retrospective database analysis task force report-part I. Value Health. 2009;12(8):1044-1052. doi:10.1111/j.1524-4733.2009.00600.x

7. Cox E, Martin BC, Van Staa T, Garbe E, Siebert U, Johnson ML. Good research practices for comparative effectiveness research: approaches to mitigate bias and confounding in the design of nonrandomized studies of treatment effects using secondary data sources: the international society for pharmacoeconomics and outcomes research good research practices for retrospective database analysis task force report-part iI. Value Health. 2009;12(8):1053-1061. doi:10.1111/j.1524-4733.2009.00601.x

8. Van Cutsem E, Kang Y, Chung H, et al. Efficacy results from the ToGA trial: a phase III study of trastuzumab added to standard chemotherapy in first-line HER2-positive advanced gastric cancer. J clin Oncol. 2009;27 (18suppl):LBA4509. doi:10.1200/jco.2009.27.18s.lba4509

9. Hess LM, Cinfio FN, Wetmore S, et al. Enhancing the budget impact model for institutional use: a tool with practical applications for the hospital oncology pharmacy. Hosp Pharm. 2016;51(6):452-460. doi:10.1310/hpj5106-452

10. Results of analyses for chemotherapy administration utilization and chemotherapy drug utilization, 2005-2011 for medicare fee-forservice beneficiaries; 2013. Available from: http://www.siteneutral. com/wp-content/uploads/2016/06/16_Final-Moran-Memo.pdf. Accessed September 23, 2019.
11. Li Q, Jiang H, Li H, et al. Efficacy of trastuzumab beyond progression in HER2 positive advanced gastric cancer: a multicenter prospective observational cohort study. Oncotarget. 2016;7(31):50656.

12. Namikawa T, Munekage E, Munekage M, et al. Evaluation of a trastuzumab-containing treatment regimen for patients with unresectable advanced or recurrent gastric cancer. Mol clin Oncol. 2016;5 (1):74-78. doi:10.3892/mco.2016.892

13. Yi JH, Kang JH, Hwang IG, et al. A retrospective analysis for patients with HER2-positive gastric cancer who were treated with trastuzumab-based chemotherapy: in the perspectives of ethnicity and histology. Cancer Res Treat. 2016;48(2):553-560. doi:10.4143/ crt.2015.155

14. Paulson AS, Hess LM, Liepa AM, et al. Ramucirumab for the treatment of patients with gastric or gastroesophageal junction cancer in community oncology practices. Gastric Cancer. 2018;21 (5):831-844. doi:10.1007/s10120-018-0796-z

15. FDA. Orphan drug designations and approvals; 2018. Available from: https://www.accessdata.fda.gov/scripts/opdlisting/oopd/index.cfm. Accessed Oct 20, 2018.

16. Ajani JA, Barthel JS, Bekaii-Saab T, et al. Gastric cancer. JNCCN. 2010;8(4):378-409. doi:10.6004/jncen.2010.0030

17. Siegel RL, Miller KD, Jemal A. Cancer statistics. CA Cancer J Clin. 2015;65(1):5-29. doi:10.3322/caac.21254

18. Gravalos C, Marquez A, Garcia-Carbonero R, et al. Correlation between HER2/neu overexpression/amplification and clinicopathological parameters in advanced gastric cancer patients: a prospective study. Gastrointestinal Cancers Symp. 2007;130(Abst):89.

19. Grillo F, Fassan M, Fiocca R, Mastracci L. Heterogeneous HER2/neu expression in gastric and gastroesophageal cancer. Human Pathol. 2016;48:173-174. doi:10.1016/j.humpath.2015.08.023

20. Hess LM, Michael D, Mytelka DS, Beyrer J, Liepa AM, Nicol S. Chemotherapy treatment patterns, costs, and outcomes of patients with gastric cancer in the United States: a retrospective analysis of electronic medical record (EMR) and administrative claims data. Gastric Cancer. 2016;19(2):607-615. doi:10.1007/s10120-0150486-z

21. Johnson ML, Crown W, Martin BC, Dormuth CR, Siebert U. Good research practices for comparative effectiveness research: analytic methods to improve causal inference from nonrandomized studies of treatment effects using secondary data sources: the ISPOR good research practices for retrospective database analysis task force report-part III. Value Health. 2009;12(8):1062-1073. doi:10.1111/ j.1524-4733.2009.00602.x
Pragmatic and Observational Research

\section{Publish your work in this journal}

Pragmatic and Observational Research is an international, peerreviewed, open access journal that publishes data from studies designed to reflect more closely medical interventions in realworld clinical practice compared with classical randomized controlled trials (RCTs). The manuscript management system is completely online and includes a very quick and fair peer-review system. Visit $\mathrm{http}: / / \mathrm{www}$.dovepress.com/testimonials.php to read real quotes from published authors. 